\title{
Regularity and Stability for a Convex Feasibility Problem
}

\author{
Carlo Alberto De Bernardi ${ }^{1}$ (D) . Enrico Miglierina ${ }^{1}$
}

Received: 23 July 2020 / Accepted: 31 July 2021 / Published online: 3 September 2021

(C) The Author(s) 2021

\begin{abstract}
Let us consider two sequences of closed convex sets $\left\{A_{n}\right\}$ and $\left\{B_{n}\right\}$ converging with respect to the Attouch-Wets convergence to $A$ and $B$, respectively. Given a starting point $a_{0}$, we consider the sequences of points obtained by projecting onto the "perturbed" sets, i.e., the sequences $\left\{a_{n}\right\}$ and $\left\{b_{n}\right\}$ defined inductively by $b_{n}=P_{B_{n}}\left(a_{n-1}\right)$ and $a_{n}=P_{A_{n}}\left(b_{n}\right)$. Suppose that $A \cap B$ is bounded, we prove that if the couple $(A, B)$ is (boundedly) regular then the couple $(A, B)$ is $d$-stable, i.e., for each $\left\{a_{n}\right\}$ and $\left\{b_{n}\right\}$ as above we have $\operatorname{dist}\left(a_{n}, A \cap B\right) \rightarrow 0$ and $\operatorname{dist}\left(b_{n}, A \cap B\right) \rightarrow 0$. Similar results are obtained also in the case $A \cap B=\emptyset$, considering the set of best approximation pairs instead of $A \cap B$.
\end{abstract}

Keywords Convex feasibility problem $\cdot$ Stability $\cdot$ Regularity $\cdot$ Set-convergence $\cdot$ Alternating projections method

Mathematics Subject Classification (2010) Primary: 47J25; secondary: 90C25 · 90C48

\section{Introduction}

Let $A$ and $B$ be two closed convex nonempty sets in a Hilbert space $X$. The (2-set) convex feasibility problem asks to find a point in the intersection of $A$ and $B$ (or, when $A \cap B=\emptyset$, a pair of points, one in $A$ and the other in $B$, that realizes the distance between $A$ and $B)$. The relevance of this problem is due to the fact that many mathematical and concrete problems in applications can be formulated as a convex feasibility problem. As typical examples, we mention solution of convex inequalities, partial differential equations, minimization of convex nonsmooth functions, medical imaging, computerized tomography and image reconstruction.

Carlo Alberto De Bernardi

carloalberto.debernardi@ unicatt.it; carloalberto.debernardi@gmail.com

Enrico Miglierina

enrico.miglierina@unicatt.it

1 Dipartimento di Matematica per le Scienze economiche, finanziarie ed attuariali, Università Cattolica del Sacro Cuore, Via Necchi 9, 20123 Milano, Italy 
The method of alternating projections is the simplest iterative procedure for finding a solution of the convex feasibility problem and it goes back to von Neumann [15]: let us denote by $P_{A}$ and $P_{B}$ the projections onto the sets $A$ and $B$, respectively, and, given a starting point $c_{0} \in X$, consider the alternating projections sequences $\left\{c_{n}\right\}$ and $\left\{d_{n}\right\}$ given by

$$
d_{n}=P_{B}\left(c_{n-1}\right) \text { and } c_{n}=P_{A}\left(d_{n}\right) \quad(n \in \mathbb{N}) .
$$

If the sequences $\left\{c_{n}\right\}$ and $\left\{d_{n}\right\}$ converge in norm, we say that the method of alternating projections converges. Originally, von Neumann proved that the method of alternating projection converges when $A$ and $B$ are closed subspaces. Then, for two generic convex sets, the weak convergence of the alternating projection sequences was proved by Bregman in 1965 [5]. Nevertheless, the problem of whether the alternating projections algorithm converges in norm for each couple of convex sets remained open till the example given by Hundal in 2004 [12]. This example shows that the alternating projections do not converge in norm when $A$ is a suitable convex cone and $B$ is a hyperplane touching the vertex of $A$. Moreover, this example emphasizes the importance of finding sufficient conditions ensuring the norm convergence of the alternating projections algorithm. In the literature, conditions of this type were studied (see, e.g., [1, 3]), even before the example by Hundal. Here, we focus on those conditions based on the notions of regularity, introduced in [1]. Indeed, in the present paper, we investigate the relationships between regularity of the couple $(A, B)$ (see Definition 3.1 below) and "stability" properties of the alternating projections method in the following sense. Let us suppose that $\left\{A_{n}\right\}$ and $\left\{B_{n}\right\}$ are two sequences of closed convex sets such that $A_{n} \rightarrow A$ and $B_{n} \rightarrow B$ for the Attouch-Wets variational convergence (see Definition 2.2) and let us introduce the definition of perturbed alternating projections sequences.

Definition 1.1 Given $a_{0} \in X$, the perturbed alternating projections sequences $\left\{a_{n}\right\}$ and $\left\{b_{n}\right\}$, w.r.t. $\left\{A_{n}\right\}$ and $\left\{B_{n}\right\}$ and with starting point $a_{0}$, are defined inductively by

$$
b_{n}=P_{B_{n}}\left(a_{n-1}\right) \text { and } a_{n}=P_{A_{n}}\left(b_{n}\right) \quad(n \in \mathbb{N}) .
$$

Our aim is to find some conditions on the limit sets $A$ and $B$ such that, for each choice of the sequences $\left\{A_{n}\right\}$ and $\left\{B_{n}\right\}$ and for each choice of the starting point $a_{0}$, the corresponding perturbed alternating projections sequences $\left\{a_{n}\right\}$ and $\left\{b_{n}\right\}$ satisfy $\operatorname{dist}\left(a_{n}, A \cap B\right) \rightarrow 0$ and $\operatorname{dist}\left(b_{n}, A \cap B\right) \rightarrow 0$. If this is the case, we say that the couple $(A, B)$ is $d$-stable. In particular, we show that the regularity of the couple $(A, B)$ implies not only the norm convergence of the alternating projections sequences for the couple $(A, B)$ (as already known from [1]), but also that the couple $(A, B)$ is $d$-stable. This result might be interesting also in applications since real data are often affected by some uncertainties. Hence stability of the convex feasibility problem with respect to data perturbations is a desirable property, also in view of computational developments.

Let us conclude the introduction by a brief description of the structure of the paper. In Section 2, we list some notations and definitions, and we recall some well-known facts about the alternating projections method. Section 3 is devoted to various notions of regularity and their relationships. It is worth pointing out that in this section we provide a new and alternative proof of the convergence of the alternating projections algorithm under regularity assumptions. This proof well illustrates the main geometrical idea behind the proof of our main result Theorem 4.9, stated and proved in Section 4. This result shows that a regular couple $(A, B)$ is d-stable whenever $A \cap B$ (or a suitable substitute if $A \cap B=\emptyset$ ) is bounded. Corollaries 4.16, 4.18, and 4.19 simplify and generalize some of the results obtained in [9], 
since there we considered only the case where $A \cap B \neq \emptyset$ whereas, in the present paper, we encompass also the situation where the intersection of $A$ and $B$ is empty. We conclude the paper with Section 5, where we discuss the necessity of the assumptions of our main result and we state a natural open problem: suppose that $A \cap B$ is bounded, is regularity equivalent to $d$-stability?

\section{Notation and Preliminaries}

Throughout all this paper, $X$ denotes a nontrivial real normed space with the topological dual $X^{*}$. We denote by $B_{X}$ and $S_{X}$ the closed unit ball and the unit sphere of $X$, respectively. If $\alpha>0, x \in X$, and $A, B \subset X$, we denote as usual

$$
x+A:=\{x+a ; a \in A\}, \quad \alpha A:=\{\alpha a ; a \in A\}, \quad A+B:=\{a+b ; a \in A, b \in B\} .
$$

For $x, y \in X,[x, y]$ denotes the closed segment in $X$ with endpoints $x$ and $y$, and $(x, y)=$ $[x, y] \backslash\{x, y\}$ is the corresponding "open" segment. For a subset $A$ of $X$, we denote by int $(A), \operatorname{conv}(A)$ and $\overline{c o n v}(A)$ the interior, the convex hull and the closed convex hull of $A$, respectively. Let us recall that a body is a closed convex set in $X$ with nonempty interior.

We denote by

$$
\operatorname{diam}(A):=\sup _{x, y \in A}\|x-y\|,
$$

the (possibly infinite) diameter of $A$. For $x \in X$, let

$$
\operatorname{dist}(x, A):=\inf _{a \in A}\|a-x\| .
$$

Moreover, given $A, B$ nonempty subsets of $X$, we denote by $\operatorname{dist}(A, B)$ the usual "distance" between $A$ and $B$, that is,

$$
\operatorname{dist}(A, B):=\inf _{a \in A} \operatorname{dist}(a, B) .
$$

Now, we recall two notions of convergence for sequences of sets (for a wide overview about this topic see, e.g., [2]). By $\mathrm{c}(X)$ we denote the family of all nonempty closed subsets of $X$. Let us introduce the (extended) Hausdorff metric $h$ on $\mathrm{c}(X)$. For $A, B \in \mathrm{c}(X)$, we define the excess of $A$ over $B$ as

$$
e(A, B):=\sup _{a \in A} \operatorname{dist}(a, B) .
$$

Moreover, if $A \neq \emptyset$ and $B=\emptyset$ we put $e(A, B)=\infty$, if $A=\emptyset$ we put $e(A, B)=0$. Then, we define

$$
h(A, B):=\max \{e(A, B), e(B, A)\} .
$$

Definition 2.1 A sequence $\left\{A_{j}\right\}$ in $\mathrm{c}(X)$ is said to Hausdorff converge to $A \in \mathrm{c}(X)$ if

$$
\lim _{j} h\left(A_{j}, A\right)=0 \text {. }
$$

As the second notion of convergence, we consider the so called Attouch-Wets convergence (see, e.g., [14, Definition 8.2.13]), which can be seen as a localization of the Hausdorff convergence. If $N \in \mathbb{N}$ and $A, B \in \mathrm{c}(X)$, define

$$
\begin{aligned}
e_{N}(A, B) & :=e\left(A \cap N B_{X}, B\right) \in[0, \infty), \\
h_{N}(A, B) & :=\max \left\{e_{N}(A, B), e_{N}(B, A)\right\} .
\end{aligned}
$$


Definition 2.2 A sequence $\left\{A_{j}\right\}$ in $\mathrm{c}(X)$ is said to Attouch-Wets converge to $A \in \mathrm{c}(X)$ if, for each $N \in \mathbb{N}$,

$$
\lim _{j} h_{N}\left(A_{j}, A\right)=0 .
$$

In the last section of our paper we shall need the following elementary fact, related to localized Hausdorff distance between two sets.

Fact 2.3 Let $D: X \rightarrow X$ be a bounded linear operator. Let $\varepsilon, \delta \in(0,1)$ and suppose that $\|D-I\| \leq \varepsilon$ and $b \in \delta B_{X}$. If $A \subset X$ and $N \in \mathbb{N}$ then

$$
h_{N}(A, b+D(A)) \leq \delta+\frac{N+\delta}{1-\varepsilon} \varepsilon .
$$

Proof If $x \in A \cap N B_{X}$ then

$$
\begin{aligned}
d(x, b+D(A)) & =\inf _{y \in D(A)}\|b+y-x\| \\
& \leq\|b+D(x)-x\| \\
& \leq\|b\|+\|D-I\|\|x\| \leq \delta+N \varepsilon .
\end{aligned}
$$

Therefore, we conclude that

$$
e_{N}(A, b+D(A))=\sup _{x \in A \cap N B_{X}} d(x, b+D(A)) \leq \delta+N \varepsilon \leq \delta+\frac{N+\delta}{1-\varepsilon} \varepsilon .
$$

Now, suppose that $x \in A$ and $y=b+D(x) \in[b+D(A)] \cap N B_{X}$, then

$$
\|x\| \leq\|x-D(x)\|+\|y\|+\|b\| \leq \varepsilon\|x\|+N+\delta,
$$

and hence $\|x\| \leq \frac{N+\delta}{1-\varepsilon}$. Proceeding as above, we have

$$
e_{N}(b+D(A), A) \leq \delta+\frac{N+\delta}{1-\varepsilon} \varepsilon .
$$

The notions of distance between two convex sets and of projection of a point onto a convex set of a Hilbert space play a fundamental role in our paper. Unless otherwise stated, from now on, $X$ will denote an Hilbert space endowed with the inner product $\langle\cdot, \cdot\rangle$. The projection onto a closed convex nonempty subset $C$ sends any point $x_{0} \in X$ to its nearest point in $C$, denoted by $P_{C}\left(x_{0}\right)$. We shall frequently use in the paper the following result, usually called variational characterization of the projection onto $C$. Let $c_{0} \in C$ and $x_{0} \in X$, then $c_{0}=P_{C}\left(x_{0}\right)$ if and only if

$$
\left\langle x_{0}-c_{0}, c-c_{0}\right\rangle \leq 0, \quad \text { whenever } c \in C .
$$

We recall that the angle $\operatorname{ang}(u, v)$ between two nonnull vectors $u, v \in X$ is defined by means of the equality

$$
\operatorname{ang}(u, v):=\arccos \left(\frac{\langle u, v\rangle}{\|u\|\|v\|}\right) .
$$

In the sequel of the paper, we denote the cosine of the angle between two nonnull vectors $u, v \in X$ as

$$
\cos (u, v):=\cos (\operatorname{ang}(u, v))=\frac{\langle u, v\rangle}{\|u\|\|v\|} .
$$

It is clear that, if $x_{0} \notin C$, (1) is equivalent to the following condition:

$$
\frac{\pi}{2} \leq \operatorname{ang}\left(x_{0}-c_{0}, c-c_{0}\right) \leq \pi, \quad \text { whenever } c \in C \backslash\left\{c_{0}\right\} .
$$


Finally, we recall that the projection $P_{C}$ is a nonexpansive map from $X$ to $C$, i.e., it holds $\left\|P_{C}(x)-P_{C}(y)\right\| \leq\|x-y\|$ (see, e.g., [14, Proposition 10.4.8]). Now, let us consider two closed convex nonempty subsets $A$ and $B$ of $X$, we denote by

$$
\begin{aligned}
& E:=\{a \in A ; d(a, B)=d(A, B)\}, \\
& F:=\{b \in B ; d(b, B)=d(A, B)\} .
\end{aligned}
$$

We say that $v:=P_{\overline{B-A}}(0)$ is the displacement vector for the couple $(A, B)$. It is clear that if $A \cap B \neq \emptyset$ then $E=F=A \cap B$ and the displacement vector for the couple $(A, B)$ is null. We recall the following fact, where, given a map $T: X \rightarrow X, \operatorname{Fix}(T)$ denotes the set of all fixed points of $T$.

Fact 2.4 ([1, Fact 1.1]) Suppose that $X$ is a Hilbert space and that A, B are closed convex nonempty subsets of $X$. Then we have:

(i) $\|v\|=\operatorname{dist}(A, B)$ and $E+v=F$;

(ii) $E=\operatorname{Fix}\left(P_{A} P_{B}\right)=A \cap(B-v)$ and $F=\operatorname{Fix}\left(P_{B} P_{A}\right)=B \cap(A+v)$;

(iii) $P_{B} e=P_{F} e=e+v(e \in E)$ and $P_{A} f=P_{E} f=f-v(f \in F)$.

We conclude this section by proving a relationship between the Attouch-Wets convergence of a sequence $\left\{A_{n}\right\}$ of closed convex sets and the convergence of the sequence $\left\{P_{A_{n}}\right\}$ of projections onto $A_{n}$ (see Lemma 2.6). This results is probably known but we were not able to find any reference in the literature, hence we provide a detailed proof for the sake of completeness. In order to prove this result we need to prove a preliminary lemma based on a geometrical property of the unit ball that holds in every Hilbert space. This property, called uniform rotundity, can be seen as a strengthening of the convexity of the unit ball and it is widely studied in the framework of the geometry of Banach space (see, e.g., [11]) Let us recall that, given a normed space $Z$, the modulus of convexity of $Z$ is the function $\delta_{Z}:[0,2] \rightarrow[0,1]$ defined by

$$
\delta_{Z}(\eta)=\inf \left\{1-\left\|\frac{x+y}{2}\right\|: x, y \in B_{X},\|x-y\| \geq \eta\right\} .
$$

It is clear that $\delta_{Z}\left(\eta_{1}\right) \leq \delta_{Z}\left(\eta_{2}\right)$, whenever $0 \leq \eta_{1} \leq \eta_{2} \leq 2$. Moreover, if $r>0$ and $\eta \in[0,2]$, by recalling the positive homogeneity of the norm, we have

$$
r \delta_{Z}\left(\frac{\eta}{r}\right)=\inf \left\{r-\left\|\frac{x+y}{2}\right\|: x, y \in r B_{X},\|x-y\| \geq \eta\right\} .
$$

In particular, if $r, M>0$ and $x, y \in r B_{X}$ are such that $\|x-y\| \geq M$ then we have

$$
\left\|\frac{x+y}{2}\right\| \leq r\left[1-\delta_{Z}\left(\frac{M}{r}\right)\right] .
$$

We say that $Z$ is uniformly rotund if $\delta_{Z}(\eta)>0$, whenever $\eta \in(0,2]$. It is well known (see, e.g., [11]) that Hilbert spaces are uniformly rotund, but there are uniformly rotund spaces that are not Hilbert spaces. Therefore, it is worth to state and prove the following lemma in this general framework. Moreover, this result, roughly speaking, says that if a convex set in a uniformly rotund space is contained in a sufficiently tight annulus between two spheres, then its diameter is as small as we want.

Lemma 2.5 Let $Z$ be a uniformly rotund normed space. Let $H, K, M>0$, then there exists $\varepsilon^{\prime} \in(0, H)$ such that, if $\rho \in[0, K]$ and if $C$ is a convex set such that $\rho-\varepsilon^{\prime} \leq\|c\| \leq \rho+\varepsilon^{\prime}$, whenever $c \in C$, then $\operatorname{diam}(C) \leq M$. 
Proof Suppose without any loss of generality that $M \leq 2$ and $H \leq 1$. We claim that any $\varepsilon^{\prime} \in(0, H)$ such that $\varepsilon^{\prime}\left[2-\delta_{Z}\left(\frac{M}{K+1}\right)\right]<\frac{M}{4} \delta_{Z}\left(\frac{M}{K+1}\right)$ works. Let $\rho \in[0, K]$ and let $C$ be a convex set such that $\rho-\varepsilon^{\prime} \leq\|c\| \leq \rho+\varepsilon^{\prime}$, whenever $c \in C$. First, observe that, since $\delta_{Z}$ assumes values in $[0,1]$, we have $\varepsilon^{\prime}<\frac{M}{4}$. Hence, if $\rho<\frac{M}{4}$, we have

$$
\operatorname{diam}(C) \leq 2\left(\rho+\varepsilon^{\prime}\right) \leq M .
$$

Now, suppose that $\rho \geq \frac{M}{4}$ and let us prove that $\operatorname{diam}(C) \leq M$. Suppose on the contrary that there exist $c_{1}, c_{2} \in C$ satisfying $\left\|c_{1}-c_{2}\right\|>M$. Put $r:=\rho+\varepsilon^{\prime}$. By (2) and since $\frac{c_{1}+c_{2}}{2} \in C$, we have

$$
\rho-\varepsilon^{\prime} \leq\left\|\frac{c_{1}+c_{2}}{2}\right\| \leq r\left[1-\delta_{Z}\left(\frac{M}{r}\right)\right] \leq r\left[1-\delta_{Z}\left(\frac{M}{K+1}\right)\right] .
$$

Therefore, we have $\varepsilon^{\prime}\left[2-\delta_{Z}\left(\frac{M}{K+1}\right)\right] \geq \rho \delta_{Z}\left(\frac{M}{K+1}\right) \geq \frac{M}{4} \delta_{Z}\left(\frac{M}{K+1}\right)$, against the definition of $\varepsilon^{\prime}$.

We are now in position to state and prove the result that links convergence of sets $A_{n}$ with that of projections onto $A_{n}$.

Lemma 2.6 Let $X$ be a Hilbert space. Suppose that a sequence $\left\{A_{n}\right\}$ in $\mathrm{c}(X)$ Attouch-Wets converges to $A \in \mathrm{c}(X)$. Then the corresponding sequence of projections $\left\{P_{A_{n}}\right\}$ uniformly converges on bounded set to $P_{A}$.

Proof Without any loss of generality we can suppose that $0 \in A$. Let us prove that, for each $K, M>0$, there exists $n_{0} \in \mathbb{N}$ such that

$$
\sup _{x \in K B_{X}}\left\|P_{A_{n}} x-P_{A} x\right\| \leq M,
$$

whenever $n \geq n_{0}$. By Lemma 2.5 (where we take $H=K$ ), there exists $\varepsilon^{\prime} \in(0, K)$ such that, if $\rho \in[0, K]$ and if $C$ is a convex set such that $\rho-\varepsilon^{\prime} \leq\|c\| \leq \rho+\varepsilon^{\prime}$, whenever $c \in C$, then $\operatorname{diam}(C) \leq M$. Since $\left\{A_{n}\right\}$ Attouch-Wets converges to $A$, there exists $n_{0} \in \mathbb{N}$ such that, for $n \geq n_{0}$, we have

(i) $A_{n} \cap 3 K B_{X} \subset A+\varepsilon^{\prime} B_{X}$;

(ii) $A \cap 3 K B_{X} \subset A_{n}+\varepsilon^{\prime} B_{X}$;

Let $x \in K B_{X}, y=P_{A} x, n \geq n_{0}$ and $y_{n}=P_{A_{n}} x$. Put $\rho=\|x-y\|$ and observe that $\rho \leq K$ since $0 \in A$. By (ii),

$$
\left\|x-y_{n}\right\| \leq\|x-y\|+\left\|y-y_{n}\right\| \leq \rho+\varepsilon^{\prime}
$$

and hence $\left\|y_{n}\right\| \leq\|x\|+\left\|x-y_{n}\right\| \leq 3 K$. Therefore, by (i), $y_{n}$ belongs to the convex set

$$
C:=\left(A+\varepsilon^{\prime} B_{X}\right) \cap\left[x+\left(\rho+\varepsilon^{\prime}\right) B_{X}\right] .
$$

Moreover, since $\operatorname{dist}(x, A)=\rho$, we have $\operatorname{dist}(x, C) \geq \rho-\varepsilon^{\prime}$. It follows that every $c \in C$ satisfies

$$
\rho-\varepsilon^{\prime} \leq\|c-x\| \leq \rho+\varepsilon^{\prime} .
$$

Hence, the assumptions of Lemma 2.5 hold for the set $C-x$ and we obtain that $\operatorname{diam}(C-$ $x)=\operatorname{diam} C \leq M$. It follows that $\left\|y_{n}-y\right\|=\left\|P_{A_{n}} x-P_{A} x\right\| \leq M$. By the arbitrariness of $x \in K B_{X}$, the proof is concluded. 


\section{Notions of Regularity for a Couple of Convex Sets}

In this section we introduce some notions of regularity for a couple of nonempty closed convex sets $A$ and $B$. This class of notions was originally introduced in [1], in order to obtain some conditions ensuring the norm convergence of the alternating projections algorithm (see, also, [4]). Here we list three different type of regularity: (i) and (ii) are exactly as they appeared in [1], whereas (iii) is new. See [1] for concrete examples of couple of sets satisfying or not properties (i) and (ii). In particular, observe that, by [1, Theorem 3.9], bounded regularity always holds when $X$ is finite-dimensional.

Definition 3.1 Let $X$ be a Hilbert space and $A, B$ closed convex nonempty subsets of $X$. Suppose that $E, F$ are nonempty. We say that the couple $(A, B)$ is:

(i) regular if for each $\varepsilon>0$ there exists $\delta>0$ such that $\operatorname{dist}(x, E) \leq \varepsilon$, whenever $x \in X$ satisfies

$$
\max \{\operatorname{dist}(x, A), \operatorname{dist}(x, B-v)\} \leq \delta ;
$$

(ii) boundedly regular if for each bounded set $S \subset X$ and for each $\varepsilon>0$ there exists $\delta>0$ such that $\operatorname{dist}(x, E) \leq \varepsilon$, whenever $x \in S$ satisfies

$$
\max \{\operatorname{dist}(x, A), \operatorname{dist}(x, B-v)\} \leq \delta ;
$$

(iii) linearly regular for points bounded away from $E$ if for each $\varepsilon>0$ there exists $K>0$ such that

$$
\operatorname{dist}(x, E) \leq K \max \{\operatorname{dist}(x, A), \operatorname{dist}(x, B-v)\},
$$

whenever $\operatorname{dist}(x, E) \geq \varepsilon$.

The following proposition shows that (i) and (iii) in the definition above are equivalent. The latter part of the proposition is a generalization of [1, Theorem 3.15].

Proposition 3.2 Let $X$ be a Hilbert space and A, B closed convex nonempty subsets of $X$. Suppose that $E, F$ are nonempty. Let us consider the following conditions.

(i) The couple $(A, B)$ is regular.

(ii) The couple $(A, B)$ is boundedly regular.

(iii) The couple $(A, B)$ is linearly regular for points bounded away from $E$.

Then $($ iii $) \Leftrightarrow(i) \Rightarrow$ (ii). Moreover, if $E$ is bounded, then $(i i) \Rightarrow(i)$.

Proof The implication $(i) \Rightarrow$ (ii) is trivial. The implication (iii) $\Rightarrow$ (i) follows directly from the definition. Indeed, by contradiction let us suppose that $(i)$ does not hold, i.e., there exist $\bar{\varepsilon}>0$ and a sequence $\left\{x_{n}\right\} \subset X$ such that $\operatorname{dist}\left(x_{n}, E\right)>\bar{\varepsilon}$ and

$$
\max \{\operatorname{dist}(x, A), \operatorname{dist}(x, B-v)\} \rightarrow 0 .
$$

By (iii) we have that $\operatorname{dist}\left(x_{n}, E\right) \rightarrow 0$, a contradiction.

Now, let us prove that $(i) \Rightarrow$ (iii). Suppose on the contrary that there exist $\varepsilon>0$ and a sequence $\left\{x_{n}\right\} \subset X$ such that $\operatorname{dist}\left(x_{n}, E\right)>\varepsilon(n \in \mathbb{N})$ and

$$
\frac{\max \left\{\operatorname{dist}\left(x_{n}, A\right), \operatorname{dist}\left(x_{n}, B-v\right)\right\}}{\operatorname{dist}\left(x_{n}, E\right)} \rightarrow 0 .
$$

For each $n \in \mathbb{N}$, let $e_{n} \in E, a_{n} \in A$, and $b_{n} \in B$ be such that $\left\|e_{n}-x_{n}\right\|=\operatorname{dist}\left(x_{n}, E\right)$, $\left\|a_{n}-x_{n}\right\|=\operatorname{dist}\left(x_{n}, A\right)$, and $\left\|b_{n}-v-x_{n}\right\|=\operatorname{dist}\left(x_{n}, B-v\right)$. Put $\lambda_{n}=\frac{\varepsilon}{\left\|e_{n}-x_{n}\right\|} \in(0,1)$ 
and define $z_{n}=\lambda_{n} x_{n}+\left(1-\lambda_{n}\right) e_{n}, a_{n}^{\prime}=\lambda_{n} a_{n}+\left(1-\lambda_{n}\right) e_{n} \in A$, and $b_{n}^{\prime}=\lambda_{n} b_{n}+(1-$ $\left.\lambda_{n}\right)\left(e_{n}+v\right) \in B$. By our construction, it is clear that

$$
\frac{\operatorname{dist}\left(z_{n}, A\right)}{\varepsilon} \leq \frac{\left\|z_{n}-a_{n}^{\prime}\right\|}{\varepsilon}=\frac{\left\|x_{n}-a_{n}\right\|}{\left\|e_{n}-x_{n}\right\|} \text { and } \frac{\operatorname{dist}\left(z_{n}, B-v\right)}{\varepsilon} \leq \frac{\left\|b_{n}^{\prime}-v-z_{n}\right\|}{\varepsilon}=\frac{\left\|b_{n}-v-x_{n}\right\|}{\left\|e_{n}-x_{n}\right\|} .
$$

Hence, $\operatorname{dist}\left(z_{n}, E\right)=\varepsilon$ and $\max \left\{\operatorname{dist}\left(z_{n}, A\right), \operatorname{dist}\left(z_{n}, B-v\right)\right\} \rightarrow 0$. This contradicts (i).

Now, suppose that $E$ is bounded, and let us prove that $(i i) \Rightarrow(i)$. Suppose on the contrary that there exist $\varepsilon>0$ and a sequence $\left\{x_{n}\right\} \subset X$ such that $\operatorname{dist}\left(x_{n}, E\right)>\varepsilon(n \in \mathbb{N})$ and

$$
\max \left\{\operatorname{dist}\left(x_{n}, A\right), \operatorname{dist}\left(x_{n}, B-v\right)\right\} \rightarrow 0 .
$$

For each $n \in \mathbb{N}$, let $e_{n} \in E, a_{n} \in A$, and $b_{n} \in B$ be such that $\left\|e_{n}-x_{n}\right\|=\operatorname{dist}\left(x_{n}, E\right)$, $\left\|a_{n}-x_{n}\right\|=\operatorname{dist}\left(x_{n}, A\right)$, and $\left\|b_{n}-v-x_{n}\right\|=\operatorname{dist}\left(x_{n}, B-v\right)$. Put $\lambda_{n}=\frac{\varepsilon}{\left\|e_{n}-x_{n}\right\|} \in(0,1)$ and define $z_{n}=\lambda_{n} x_{n}+\left(1-\lambda_{n}\right) e_{n}, a_{n}^{\prime}=\lambda_{n} a_{n}+\left(1-\lambda_{n}\right) e_{n} \in A$, and $b_{n}^{\prime}=\lambda_{n} b_{n}+(1-$ $\left.\lambda_{n}\right)\left(e_{n}+v\right) \in B$. By our construction, it holds that

$$
\operatorname{dist}\left(z_{n}, A\right) \leq\left\|z_{n}-a_{n}^{\prime}\right\| \leq\left\|x_{n}-a_{n}\right\|
$$

and

$$
\operatorname{dist}\left(z_{n}, B-v\right) \leq\left\|b_{n}^{\prime}-v-z_{n}\right\| \leq\left\|b_{n}-v-x_{n}\right\| .
$$

Hence, $\operatorname{dist}\left(z_{n}, E\right)=\varepsilon$ and $\max \left\{\operatorname{dist}\left(z_{n}, A\right), \operatorname{dist}\left(z_{n}, B-v\right)\right\} \rightarrow 0$. Moreover, since $E$ is bounded $\left\{z_{n}\right\}$ is a bounded sequence. This contradicts (ii) and the proof is concluded.

The following theorem follows by [1, Theorem 3.7].

Theorem 3.3 Let $X$ be a Hilbert space and $A, B$ closed convex nonempty subsets of $X$. Suppose that the couple $(A, B)$ is regular. Then the alternating projections method converges.

We present here below a proof of this theorem, slightly different from that contained in [1]. This proof is based on a simplified version of the argument that we will use in our main result Theorem 4.9. Let us point out that this proof is not essential for sequel of the paper, but it can be useful to visualize the geometrical idea behind the proof of Theorem 4.9. We consider only the case where $A \cap B$ is nonempty since the general case is similar but some unavoidable details would have made it more difficult to follow the outline of the proof.

Proof Let us consider the sequences $\left\{c_{n}=P_{A}\left(d_{n}\right)\right\}$ and $\left\{d_{n+1}=P_{B}\left(c_{n}\right)\right\}$. By the nonexpansivity of the projections onto convex sets, for every $h \in A \cap B$, we have:

$$
\begin{gathered}
\left\|c_{n}-h\right\|=\left\|P_{A}\left(d_{n}\right)-P_{A}(h)\right\| \leq\left\|d_{n}-h\right\| \\
\left\|d_{n+1}-h\right\|=\left\|P_{B}\left(c_{n}\right)-P_{B}(h)\right\| \leq\left\|c_{n}-h\right\|
\end{gathered}
$$

It follows immediately that:

( $\alpha) \quad \operatorname{dist}\left(c_{n}, A \cap B\right) \leq \operatorname{dist}\left(d_{n}, A \cap B\right)$ and $\operatorname{dist}\left(d_{n+1}, A \cap B\right) \leq \operatorname{dist}\left(c_{n}, A \cap B\right)$,

whenever $n \in \mathbb{N}$. This condition implies that [1, Theorem 3.3, (iv)] holds, therefore the following fact holds: "The sequence $\left\{c_{n}\right\}$ converges to a point in $A \cap B$ iff $\operatorname{dist}\left(c_{n}, A \cap B\right) \rightarrow$ $0 . "$ Hence, it is sufficient to prove that $\operatorname{dist}\left(c_{n}, A \cap B\right) \rightarrow 0$.

For $\varepsilon>0$, by the equivalence $(i) \Leftrightarrow$ (iii) in Proposition 3.2, there exists $K>0$ such that

$$
\operatorname{dist}(x, A \cap B) \leq K \max \{\operatorname{dist}(x, A), \operatorname{dist}(x, B)\},
$$


whenever $\operatorname{dist}(x, A \cap B) \geq \varepsilon$. Observe that $K \geq 1$ and define $\eta=\sqrt{1-\frac{1}{K^{2}}}$. We claim that, for each $n \in \mathbb{N}$, the following condition holds:

$(\beta) \quad$ if $\operatorname{dist}\left(c_{n}, A \cap B\right) \geq \varepsilon$ then $\operatorname{dist}\left(c_{n}, A \cap B\right) \leq \eta \operatorname{dist}\left(d_{n}, A \cap B\right)$.

To prove this, let $h_{n}:=P_{A \cap B}\left(d_{n}\right)$ and observe that

$$
\left[\operatorname{dist}\left(c_{n}, A \cap B\right)\right]^{2}+\left[\operatorname{dist}\left(d_{n}, A\right)\right]^{2} \leq\left\|c_{n}-h_{n}\right\|^{2}+\left[\operatorname{dist}\left(d_{n}, A\right)\right]^{2} .
$$

By the variational characterization of the projection and since $c_{n}=P_{A}\left(d_{n}\right)$, we obtain that the angle $\theta_{n}:=\operatorname{ang}\left(d_{n}-c_{n}, h_{n}-c_{n}\right)$ is such that

$$
\frac{\pi}{2} \leq \theta_{n} \leq \pi \text {. }
$$

Hence, (4) implies

$$
\begin{aligned}
{\left[\operatorname{dist}\left(c_{n}, A \cap B\right)\right]^{2}+\left[\operatorname{dist}\left(d_{n}, A\right)\right]^{2} \leq } & \left\|c_{n}-h_{n}\right\|^{2}+\left[\operatorname{dist}\left(d_{n}, A\right)\right]^{2} \\
& -2\left\|c_{n}-h_{n}\right\| \operatorname{dist}\left(d_{n}, A\right) \cos \theta_{n} \\
= & {\left[\operatorname{dist}\left(d_{n}, A \cap B\right)\right]^{2} }
\end{aligned}
$$

where the last equality is obtained by applying the law of cosines to the triangle with vertices $d_{n}, c_{n}$ and and $h_{n}$. This gives

$$
\left[\operatorname{dist}\left(c_{n}, A \cap B\right)\right]^{2} \leq\left[\operatorname{dist}\left(d_{n}, A \cap B\right)\right]^{2}-\left[\operatorname{dist}\left(d_{n}, A\right)\right]^{2}
$$

Finally, since, by $(\alpha), \operatorname{dist}\left(d_{n}, A \cap B\right) \geq \operatorname{dist}\left(c_{n}, A\right) \geq \varepsilon$, (3) and the last inequality gives

$$
\left[\operatorname{dist}\left(c_{n}, A \cap B\right)\right]^{2} \leq\left[\operatorname{dist}\left(d_{n}, A \cap B\right)\right]^{2}-\frac{1}{K^{2}}\left[\operatorname{dist}\left(d_{n}, A \cap B\right)\right]^{2},
$$

and the claim is proved.

Now, if there exists $n_{0} \in \mathbb{N}$ such that $\operatorname{dist}\left(c_{n_{0}}, A \cap B\right) \leq \varepsilon$, then, $(\alpha) \operatorname{implies} \operatorname{dist}\left(c_{n}, A \cap\right.$ $B) \leq \varepsilon$ for every $n \geq n_{0}$. On the other hand, if $\operatorname{dist}\left(c_{n}, A \cap B\right) \geq \varepsilon$ for all $n \in \mathbb{N}$, then, by combining subsequently $(\beta)$ and $(\alpha)$ we obtain

$$
\operatorname{dist}\left(c_{n}, A \cap B\right) \leq \eta \operatorname{dist}\left(d_{n}, A \cap B\right) \leq \eta \operatorname{dist}\left(c_{n-1}, A \cap B\right) \quad(n \in \mathbb{N}),
$$

a contradiction since $\eta<1$. Therefore we conclude that eventually $\operatorname{dist}\left(c_{n}, A \cap B\right) \leq \varepsilon$. By the arbitrariness of $\varepsilon>0$ the proof is concluded.

\section{Regularity and Perturbed Alternating Projections}

This section is devoted to prove our main result. Indeed, here we show that if a couple $(A, B)$ of convex closed sets is regular then not only the alternating projections method converges but also the couple $(A, B)$ satisfies certain "stability" properties with respect to perturbed projections sequences.

Let us start by making precise the word "stability" by introducing the following two notions of stability for a couple $(A, B)$ of convex closed subsets of $X$.

Definition 4.1 Let $A$ and $B$ be closed convex subsets of $X$ such that $E, F$ are nonempty. We say that the couple $(A, B)$ is stable $[d$-stable, respectively] if for each choice of sequences $\left\{A_{n}\right\},\left\{B_{n}\right\} \subset \mathrm{c}(X)$ converging with respect to the Attouch-Wets convergence to $A$ and $B$, respectively, and for each choice of the starting point $a_{0}$, the corresponding perturbed alternating projections sequences $\left\{a_{n}\right\}$ and $\left\{b_{n}\right\}$ converge in norm [satisfy $\operatorname{dist}\left(a_{n}, E\right) \rightarrow 0$ and $\operatorname{dist}\left(b_{n}, F\right) \rightarrow 0$, respectively]. 
Remark 4.2 We remark that the couple $(A, B)$ is stable if and only if for each choice of sequences $\left\{A_{n}\right\},\left\{B_{n}\right\} \subset \mathrm{c}(X)$ converging with respect to the Attouch-Wets convergence to $A$ and $B$, respectively, and for each choice of the starting point $a_{0}$, there exists $e \in E$ such that the perturbed alternating projections sequences $\left\{a_{n}\right\}$ and $\left\{b_{n}\right\}$ satisfy $a_{n} \rightarrow e$ and $b_{n} \rightarrow e+v$ in norm.

Proof Without any loss of generality, we can suppose that $0 \in B$. Let us start by proving that if $a_{n} \rightarrow e$ then $e \in E$.

We claim that the sequence $\left\{P_{A_{n}} P_{B_{n}}\right\}$ uniformly converges on the bounded sets to $P_{A} P_{B}$. To see this observe that:

- $\quad$ since $0 \in B$, we have $\left\|P_{B} x\right\| \leq\|x\|$, whenever $x \in X$;

- $\quad$ since projections are nonexpansive, we have

$$
\left\|P_{A_{n}} P_{B_{n}} x-P_{A_{n}} P_{B} x\right\| \leq\left\|P_{B_{n}} x-P_{B} x\right\|,
$$

whenever $x \in X$ and $n \in \mathbb{N}$;

- $\quad$ for each $x \in X$ and $n \in \mathbb{N}$, we have

$$
\left\|P_{A_{n}} P_{B_{n}} x-P_{A} P_{B} x\right\| \leq\left\|P_{A_{n}} P_{B_{n}} x-P_{A_{n}} P_{B} x\right\|+\left\|P_{A_{n}} P_{B} x-P_{A} P_{B} x\right\| .
$$

The previous observation implies that, for $N>0$, we have

$$
\begin{aligned}
\sup _{\|x\| \leq N}\left\|P_{A_{n}} P_{B_{n}} x-P_{A} P_{B} x\right\| \leq & \sup _{\|x\| \leq N}\left\|P_{B_{n}} x-P_{B} x\right\| \\
& +\sup _{\|x\| \leq N}\left\|P_{A_{n}} P_{B} x-P_{A} P_{B} x\right\| \\
\leq & \sup _{\|x\| \leq N}\left\|P_{B_{n}} x-P_{B} x\right\|+\sup _{\|y\| \leq N}\left\|P_{A_{n}} y-P_{A} y\right\| .
\end{aligned}
$$

Since $A_{n} \rightarrow A, B_{n} \rightarrow B$ for the Attouch-Wets convergence, by Lemma 2.6, $\left\{P_{A_{n}}\right\}$ uniformly converges on bounded set to $P_{A}$ and $\left\{P_{B_{n}}\right\}$ uniformly converges on bounded set to $P_{B}$. The claim follows by the previous inequality.

Now, since $\left\{a_{n}\right\}$ is bounded and

$$
a_{n+1}=P_{A_{n}} P_{B_{n}} a_{n}=P_{A} P_{B} a_{n}+\left(P_{A_{n}} P_{B_{n}}-P_{A} P_{B}\right) a_{n},
$$

passing to the limit as $n \rightarrow \infty$, we obtain $e=P_{A} P_{B} e$. By Fact 2.4, (ii), we have that $e \in E$. Similarly, it is easy to see that

$$
b_{n+1}=P_{B_{n}} a_{n}=P_{B} a_{n}+\left(P_{B_{n}}-P_{B}\right) a_{n} \rightarrow P_{B} e=e+v,
$$

and the proof is concluded.

It is clear that if the couple $(A, B)$ is stable, then it is $d$-stable. Moreover, if $E, F$ are singletons then also the converse implication holds true. The following basic assumptions will be considered in the sequel of the paper.

Basic assumptions 4.3 Let $A, B$ be closed convex nonempty subsets of $X$. Suppose that:

(i) $E, F$ are nonempty and bounded;

(ii) $\left\{A_{n}\right\}$ and $\left\{B_{n}\right\}$ are sequences of closed convex sets such that $A_{n} \rightarrow A$ and $B_{n} \rightarrow B$ for the Attouch-Wets convergence.

Now, let us prove a chain of lemmas and propositions that we shall use in the proof of our main result, Theorem 4.9 below. 
Lemma 4.4 Let $G$ be a closed convex subset of $X$. Suppose that there exist $\varepsilon, K>0$ such that $\varepsilon B_{X} \subset G \subset K B_{X}$. Then, if $u, w \in \partial G$ and $\theta:=\cos (u, w)>0$, we have

$$
\|u-w\|^{2} \leq K^{2}\left(\frac{K^{2}}{\varepsilon^{2}}+1\right) \frac{1-\theta^{2}}{\theta^{2}} .
$$

Proof The proof involves only the plane containing the origin and the vectors $u$ and $v$. Henceforth, without any loss of generality we can suppose that $X=\mathbb{R}^{2}$ and $u=(\|u\|, 0)$. Let us denote $w=(x, y)$, with $x, y \in \mathbb{R}$, and suppose that $u, w \in \partial G$. Observe that, since $\theta>0, x$ is positive.

We claim that it holds

$$
|y| \geq\left|\frac{\varepsilon}{\|u\|}(x-\|u\|)\right| .
$$

To prove our claim, suppose on the contrary that

$$
|y|<\left|\frac{\varepsilon}{\|u\|}(x-\|u\|)\right| .
$$

and let us consider two cases. First, let $x$ be such that $0<x \leq\|u\|$. Since $\varepsilon B_{X} \subset G$ and $u=(\|u\|, 0) \in G$, the set

$$
L:=\left\{(z, v) \in \mathbb{R}^{2} ; 0<z<\|u\|,|v|<\left|\frac{\varepsilon}{\|u\|}(z-\|u\|)\right|\right\}
$$

is contained in the interior of $G$ and $w \in L$, a contradiction. We now turn to the case $x>\|u\|$. Let $h:=\left(0,-\frac{\|u\|}{x-\|u\|} y\right) \in X$, then it holds

$$
u=\frac{\|u\|}{x} w+\left(1-\frac{\|u\|}{x}\right) h .
$$

By (6), we have $\|h\|<\varepsilon$. Then $u$ belongs to the interior of the set conv $\left(\{w\} \cup \varepsilon B_{X}\right)$, and hence to the interior of $G$. This contradiction proves the claim.

Since $\theta=\cos (u, w)=\frac{x}{\|w\|}$, we have $y^{2}=\frac{1-\theta^{2}}{\theta^{2}} x^{2}$. Hence, by (5), we have

$$
(x-\|u\|)^{2} \leq \frac{1-\theta^{2}}{\theta^{2}} \frac{\|u\|^{2}}{\varepsilon^{2}} x^{2} .
$$

Finally,

$$
\|u-w\|^{2}=(x-\|u\|)^{2}+y^{2} \leq x^{2}\left(\frac{\|u\|^{2}}{\varepsilon^{2}}+1\right) \frac{1-\theta^{2}}{\theta^{2}} \leq K^{2}\left(\frac{K^{2}}{\varepsilon^{2}}+1\right) \frac{1-\theta^{2}}{\theta^{2}} .
$$

Proposition 4.5 Let Basic assumptions 4.3 be satisfied and, for each $n \in \mathbb{N}$, let $a_{n} \in A_{n}$ and $b_{n} \in B_{n}$. Suppose that the couple $(A, B)$ is regular. Let $\varepsilon>0$, then there exist $\eta \in(0,1)$ and $n_{1} \in \mathbb{N}$ such that for each $n \geq n_{1}$ we have:

(i) if $\operatorname{dist}\left(a_{n}, E\right) \geq 2 \varepsilon$ and $\operatorname{dist}\left(b_{n}, F\right) \geq 2 \varepsilon$ then $\cos \left(a_{n}-e, b_{n}-(e+v)\right) \leq \eta$, whenever $e \in E+\varepsilon B_{X}$.

(ii) if $\operatorname{dist}\left(a_{n}, E\right) \geq 2 \varepsilon$ and $\operatorname{dist}\left(b_{n+1}, F\right) \geq 2 \varepsilon$ then $\cos \left(b_{n+1}-f, a_{n}+v-f\right) \leq \eta$, whenever $f \in F+\varepsilon B_{X}$.

Proof Let us prove that there exist $\eta \in(0,1)$ such that eventually (i) holds, the proof that there exist $\eta \in(0,1)$ such that eventually (ii) holds is similar. Suppose that this is not the 
case, then there exist sequences $\left\{e_{k}\right\} \subset E+\varepsilon B_{X},\left\{\theta_{k}\right\} \subset(0,1)$ and an increasing sequence of the integers $\left\{n_{k}\right\}$ such that $\operatorname{dist}\left(a_{n_{k}}, E\right) \geq 2 \varepsilon$, $\operatorname{dist}\left(b_{n_{k}}, F\right) \geq 2 \varepsilon$, and

$$
\cos \left(a_{n_{k}}-e_{k}, b_{n_{k}}-\left(e_{k}+v\right)\right)=\theta_{k} \rightarrow 1 \text {. }
$$

Let $G=E+2 \varepsilon B_{X}$ and observe that $G$ is a bounded body in $X$. Since $e_{k} \in \operatorname{int} G$ and $a_{n_{k}} \notin$ int $G$, there exists a unique point $a_{k}^{\prime} \in\left[e_{k}, a_{n_{k}}\right] \cap \partial G$. Similarly, there exists a unique point $b_{k}^{\prime} \in\left[e_{k}, b_{n_{k}}-v\right] \cap \partial G$. Moreover, by construction, we have that

$$
\cos \left(a_{k}^{\prime}-e_{k}, b_{k}^{\prime}-e_{k}\right)=\theta_{k} .
$$

Lemma 4.4 implies that $\left\|\left(a_{k}^{\prime}-v\right)-\left(b_{k}^{\prime}-v\right)\right\|=\left\|a_{k}^{\prime}-b_{k}^{\prime}\right\| \rightarrow 0$. Since $G$ is bounded and $A_{n_{k}} \rightarrow A, B_{n_{k}} \rightarrow B$ for the Attouch-Wets convergence, there exist sequences $\left\{a_{k}^{\prime \prime}\right\} \subset A$ and $\left\{b_{k}^{\prime \prime}\right\} \subset B-v$ such that $\left\|a_{k}^{\prime \prime}-a_{k}^{\prime}\right\| \rightarrow 0$ and $\left\|b_{k}^{\prime \prime}-b_{k}^{\prime}\right\| \rightarrow 0$. Hence, by the triangle inequality, $\left\|a_{k}^{\prime \prime}-b_{k}^{\prime \prime}\right\| \rightarrow 0$ and eventually $\operatorname{dist}\left(a_{k}^{\prime \prime}, E\right) \geq \varepsilon$, a contradiction since the couple $(A, B)$ is regular.

Proposition 4.6 Let Basic assumptions 4.3 be satisfied, suppose that the couple $(A, B)$ is regular, and let $\delta, \varepsilon>0$. For each $n \in \mathbb{N}$, let $a_{n}, x_{n} \in A_{n}$ and $b_{n}, y_{n} \in B_{n}$ be such that $\operatorname{dist}\left(x_{n}, E\right) \rightarrow 0$ and $\operatorname{dist}\left(y_{n}, F\right) \rightarrow 0$. Then there exists $n_{2} \in \mathbb{N}$ such that for each $n \geq n_{2}$ we have:

(i) if $\operatorname{dist}\left(a_{n}, E\right) \geq 2 \varepsilon$, $\operatorname{dist}\left(b_{n}, F\right) \geq 2 \varepsilon$, and $a_{n}=P_{A_{n}} b_{n}$ then

$$
\cos \left(x_{n}-a_{n}, b_{n}-\left(a_{n}+v\right)\right) \leq \delta ;
$$

(ii) if $\operatorname{dist}\left(a_{n}, E\right) \geq 2 \varepsilon$, $\operatorname{dist}\left(b_{n+1}, F\right) \geq 2 \varepsilon$, and $b_{n+1}=P_{B_{n+1}} a_{n}$ then

$$
\cos \left(y_{n+1}-b_{n+1}, a_{n}+v-b_{n+1}\right) \leq \delta \text {. }
$$

Proof Let us prove that eventually (i) holds, the proof that eventually (ii) holds is similar. Since $\operatorname{dist}\left(a_{n}, E\right) \geq 2 \varepsilon$ and $\operatorname{dist}\left(x_{n}, E\right) \rightarrow 0$ we have that eventually $x_{n}-a_{n} \neq 0$. By Proposition 4.5 , there exists $\eta \in(0,1)$ and $n_{1} \in \mathbb{N}$ such that

$$
\cos \left(a_{n}-e, b_{n}-(e+v)\right) \leq \eta,
$$

whenever $n \geq n_{1}$ and $e \in E+\varepsilon B_{X}$. Observe that, if $\operatorname{dist}\left(a_{n}, E\right) \geq 2 \varepsilon$ and $\operatorname{dist}\left(b_{n}, F\right) \geq 2 \varepsilon$, we have that $\left\|a_{n}-e\right\| \geq \varepsilon$ and $\left\|b_{n}-(e+v)\right\| \geq \varepsilon(n \in \mathbb{N})$. By the law of cosines we have $\|u-w\|^{2}=\|u\|^{2}+\|w\|^{2}-2 \cos (u, w)\|u\|\|w\| \geq 2\|u\|\|w\|(1-\cos (u, w)), \quad u, w \in X$.

By (7) and the previous inequality, applied to $w=b_{n}-(e+v)$ and $u=a_{n}-e$, there exists a constant $\eta^{\prime}>0$ such that $\left\|b_{n}-\left(a_{n}+v\right)\right\| \geq \eta^{\prime}$, whenever $n \geq n_{1}$. By the above, $x_{n}-a_{n} \neq 0$ and $b_{n}-\left(a_{n}+v\right) \neq 0$ for all $n \geq n_{1}$, then eventually $\cos \left(x_{n}-a_{n}, b_{n}-\left(a_{n}+v\right)\right)$ is well-defined. If $v=0$, the thesis is trivial since, by the variational characterization of $a_{n}=P_{A_{n}} b_{n}$, it holds

$$
\left\langle x_{n}-a_{n}, b_{n}-a_{n}\right\rangle \leq 0,
$$

whenever $n \in \mathbb{N}$.

Suppose that $v \neq 0$. We claim that, if $v$ denotes the displacement vector for the couple $(A, B)$, eventually we have

$$
\left\langle v, a_{n}-x_{n}\right\rangle \leq \delta \eta^{\prime}\left\|a_{n}-x_{n}\right\| .
$$

To prove our claim observe that, $\operatorname{since} \operatorname{dist}\left(x_{n}, E\right) \rightarrow 0$, we can suppose without any loss of generality that $\operatorname{dist}\left(x_{n}, E\right) \leq \varepsilon(n \in \mathbb{N})$. Moreover, we can consider a sequence $\left\{x_{n}^{\prime}\right\} \subset E$ such that $\left\|x_{n}^{\prime}-x_{n}\right\| \rightarrow 0$. Let $G=E+2 \varepsilon B_{X}$ and observe that $G$ is a bounded body in $X$. 
Since $x_{n} \in \operatorname{int} G$ and $a_{n} \notin$ int $G$, there exists a unique point $a_{n}^{\prime} \in\left[x_{n}, a_{n}\right] \cap \partial G$. Since $G$ is bounded and $A_{n} \rightarrow A$ for the Attouch-Wets convergence, there exists a sequence $\left\{a_{n}^{\prime \prime}\right\} \subset A$ such that $\left\|a_{n}^{\prime \prime}-a_{n}^{\prime}\right\| \rightarrow 0$. Since $\left\{x_{n}^{\prime}\right\} \subset E$, it follows that $\left\|a_{n}^{\prime \prime}-x_{n}^{\prime}\right\| \geq 2 \varepsilon$ and, by taking into account the variational characterization of the projection, that $\left\langle v, a_{n}^{\prime \prime}-x_{n}^{\prime}\right\rangle \leq 0$. Hence, eventually we have

$$
\left\langle v, a_{n}^{\prime \prime}-x_{n}^{\prime}\right\rangle-\delta \eta^{\prime}\left\|a_{n}^{\prime \prime}-x_{n}^{\prime}\right\| \leq-\delta \eta^{\prime} \varepsilon .
$$

Since $\left\|x_{n}^{\prime}-x_{n}\right\| \rightarrow 0$ and $\left\|a_{n}^{\prime \prime}-a_{n}^{\prime}\right\| \rightarrow 0$, eventually we have

$$
\left\langle v, a_{n}^{\prime}-x_{n}\right\rangle-\delta \eta^{\prime}\left\|a_{n}^{\prime}-x_{n}\right\| \leq 0 .
$$

By homogeneity of $\langle v, \cdot\rangle$ and of the norm, and by our construction, the claim is proved.

Now, by our claim, since $a_{n}=P_{A_{n}} b_{n}$ and $x_{n} \in A_{n}(n \in \mathbb{N})$, we have

$$
\left\langle x_{n}-a_{n}, b_{n}-\left(a_{n}+v\right)\right\rangle=\left\langle x_{n}-a_{n}, b_{n}-a_{n}\right\rangle+\left\langle a_{n}-x_{n}, v\right\rangle \leq \delta \eta^{\prime}\left\|a_{n}-x_{n}\right\| .
$$

Eventually, since $\left\|b_{n}-\left(a_{n}+v\right)\right\| \geq \eta^{\prime}$, we have

$$
\cos \left(x_{n}-a_{n}, b_{n}-\left(a_{n}+v\right)\right) \leq \frac{\delta \eta^{\prime}}{\left\|b_{n}-\left(a_{n}+v\right)\right\|} \leq \delta .
$$

Now, we need a simple geometrical result whose proof is a simple application of the definition of cosine combined with the triangle inequality.

Fact 4.7 Let $\eta, \eta^{\prime} \in(0,1)$ be such that $\eta<\eta^{\prime}$. If $\delta \in(0,1)$ satisfies $\frac{\delta+\eta}{1-\delta} \leq \eta^{\prime}$ and if $x, y \in X$ are linearly independent vectors such that $\cos (x, y) \leq \eta$ and $\cos (y-x,-x) \leq \delta$ then $\|x\| \leq \eta^{\prime}\|y\|$.

Proof By our hypotheses and the definition of cosine, we have

- $\quad-\langle y, x\rangle \geq-\eta\|x\|\|y\|$;

- $\quad-\langle y, x\rangle+\|x\|^{2} \leq \delta\|y-x\|\|x\|$.

Combining the two inequalities, we obtain

$$
\|x\|(\|x\|-\eta\|y\|) \leq \delta\|y-x\|\|x\|,
$$

and hence, by the triangular inequality,

$$
\|x\|-\eta\|y\| \leq \delta\|y-x\| \leq \delta\|y\|+\delta\|x\| .
$$

Finally,

$$
\|x\| \leq \frac{\delta+\eta}{1-\delta}\|y\| \leq \eta^{\prime}\|y\| .
$$

Proposition 4.8 Let Basic assumptions 4.3 be satisfied. For each $M>0$ there exist $\theta \in$ $(0, M)$ and $n_{0} \in \mathbb{N}$ such that if $n \geq n_{0}$ we have:

(i) if $b_{n} \in B_{n}, a_{n}=P_{A_{n}} b_{n}$, and $\operatorname{dist}\left(b_{n}, F\right) \leq \theta$ then

$$
\operatorname{dist}\left(a_{n}, E\right) \leq 2 M \text {; }
$$

(ii) if $a_{n} \in A_{n}, b_{n+1}=P_{B_{n+1}} a_{n}$, and $\operatorname{dist}\left(a_{n}, E\right) \leq \theta$ then

$$
\operatorname{dist}\left(b_{n+1}, F\right) \leq 2 M \text {. }
$$


Proof Let $M>0$ and $\rho=\|v\|$, where $v$ is the displacement vector. By Lemma 2.5 (where we take $H=3 M)$, there exists $\varepsilon^{\prime} \in(0,3 M)$ such that, if $C$ is a convex set such that $\rho-\varepsilon^{\prime} \leq\|c\| \leq \rho+\varepsilon^{\prime}$, whenever $c \in C$, then $\operatorname{diam}(C) \leq M$. Put $\theta=\varepsilon^{\prime} / 3$, since Basic assumption 4.3 are satisfied, there exists $n_{0} \in \mathbb{N}$ such that if $n \geq n_{0}$ we have:

(a) if $w \in A_{n}$ then $\operatorname{dist}(w, F) \geq \rho-3 \theta$;

(b) if $e \in E$, there exists $x \in A_{n}$ such that $\|e-x\| \leq \theta$.

Now, let $n \geq n_{0}, b_{n} \in B_{n}, a_{n}=P_{A_{n}} b_{n}$, and $\operatorname{dist}\left(b_{n}, F\right) \leq \theta$. Let $f_{n} \in F$ be such that $\left\|f_{n}-b_{n}\right\| \leq \theta$ and put $e_{n}=f_{n}-v \in E$. By (b), there exists $x_{n} \in A_{n}$ such that $\left\|x_{n}-e_{n}\right\| \leq \theta$. Hence, since $a_{n}=P_{A_{n}} b_{n}$ and $\left\|e_{n}-f_{n}\right\|=\rho$, we have

$$
\begin{aligned}
\left\|a_{n}-f_{n}\right\| & \leq\left\|a_{n}-b_{n}\right\|+\left\|f_{n}-b_{n}\right\| \\
& \leq\left\|x_{n}-b_{n}\right\|+\left\|f_{n}-b_{n}\right\| \\
& =\left\|x_{n}-e_{n}+e_{n}-f_{n}+f_{n}-b_{n}\right\|+\left\|f_{n}-b_{n}\right\| \\
& \leq\left\|x_{n}-e_{n}\right\|+\rho+2\left\|f_{n}-b_{n}\right\| \leq \rho+3 \theta .
\end{aligned}
$$

Let us consider the convex set $C=\left[x_{n}-f_{n}, a_{n}-f_{n}\right]$. Observe that, since

$$
\left\|x_{n}-f_{n}\right\| \leq\left\|e_{n}-x_{n}\right\|+\left\|e_{n}-f_{n}\right\| \leq \rho+\theta,
$$

we have that $\|c\| \leq \rho+3 \theta$, whenever $c \in C$. Moreover, since $\left[x_{n}, a_{n}\right] \subset A_{n}$ and $f_{n} \in F$, by (a) we have $\|c\| \geq \rho-3 \theta$, whenever $c \in C$. Hence, we can apply Lemma 2.5 to the set $C$ and we have $\left\|a_{n}-x_{n}\right\|=\operatorname{diam}(C) \leq M$. Then

$$
\operatorname{dist}\left(a_{n}, E\right) \leq\left\|a_{n}-e_{n}\right\| \leq\left\|a_{n}-x_{n}\right\|+\left\|e_{n}-x_{n}\right\| \leq M+\theta \leq 2 M .
$$

The proof that eventually (ii) holds is similar.

We are now ready to state and prove the main result of this paper.

Theorem 4.9 Let $A, B$ be closed convex nonempty subsets of $X$ such that $E$ and $F$ are bounded. Suppose that the couple $(A, B)$ is regular, then the couple $(A, B)$ is d-stable.

Proof Let $a_{0} \in X$ and let $\left\{a_{n}\right\}$ and $\left\{b_{n}\right\}$ be the corresponding perturbed alternating projections sequences, i.e,

$$
a_{n}=P_{A_{n}}\left(b_{n}\right) \quad \text { and } \quad b_{n}=P_{B_{n}}\left(a_{n-1}\right) .
$$

First of all, we remark that it is enough to prove that $\operatorname{dist}\left(a_{n}, E\right) \rightarrow 0$ since the proof that $\operatorname{dist}\left(b_{n}, F\right) \rightarrow 0$ follows by the symmetry of the problem. Therefore our aim is to prove that for each $M>0$, eventually we have

$$
\operatorname{dist}\left(a_{n}, E\right) \leq M \text {. }
$$

Let us consider $M>0$, then, by (ii) in Proposition 4.8, there exist $0<\theta<\frac{M}{2}$ and $n^{\prime} \in \mathbb{N}$ such that, for each $n>n^{\prime}$, if $\operatorname{dist}\left(a_{n}, E\right) \leq \theta$ then $\operatorname{dist}\left(b_{n+1}, F\right) \leq M$. Now, by (i) in Proposition 4.8, there exist $0<\varepsilon<\frac{\theta}{4}$ and $n^{\prime \prime} \in \mathbb{N}$ such that, for each $n>n^{\prime \prime}$, if $\operatorname{dist}\left(b_{n}, F\right) \leq 2 \varepsilon$ then $\operatorname{dist}\left(a_{n}, E\right) \leq \theta$. Therefore, we conclude that there exist $0<\varepsilon<\frac{M}{8}$ and $n_{0}=\max \left\{n^{\prime}, n^{\prime \prime}\right\} \in \mathbb{N}$ such that, for each $n>n_{0}$ we have:

$\left(\alpha_{1}\right) \quad$ if $\operatorname{dist}\left(b_{n}, F\right) \leq 2 \varepsilon$ then $\operatorname{dist}\left(a_{n}, E\right) \leq M$ and $\operatorname{dist}\left(b_{n+1}, F\right) \leq M$.

Again, by applying Proposition 4.8 twice and a similar reasoning as above, we can suppose that, for each $n \geq n_{0}$ : 
$\left(\alpha_{2}\right) \quad$ if $\operatorname{dist}\left(a_{n}, E\right) \leq 2 \varepsilon$ then $\operatorname{dist}\left(b_{n+1}, F\right) \leq M$ and $\operatorname{dist}\left(a_{n+1}, E\right) \leq M$.

Now, by Proposition 4.5 there exist $\eta \in(0,1)$ and $n_{1} \geq n_{0}$ such that:

(i) if $\operatorname{dist}\left(a_{n}, E\right) \geq 2 \varepsilon$ and $\operatorname{dist}\left(b_{n}, F\right) \geq 2 \varepsilon$ then

$$
\cos \left(a_{n}-e, b_{n}-(e+v)\right) \leq \eta,
$$

whenever $n \geq n_{1}$ and $e \in E+\varepsilon B_{X}$;

(ii) if $\operatorname{dist}\left(a_{n}, E\right) \geq 2 \varepsilon$ and $\operatorname{dist}\left(b_{n+1}, F\right) \geq 2 \varepsilon$ then

$$
\cos \left(b_{n+1}-f, a_{n}-(f-v)\right) \leq \eta,
$$

whenever $n \geq n_{1}$ and $f \in F+\varepsilon B_{X}$.

For each $n \in \mathbb{N}$, let $e_{n} \in E$ and $f_{n} \in F$ be such that $\left\|a_{n}-e_{n}\right\|=\operatorname{dist}\left(a_{n}, E\right)$ and $\left\|b_{n}-f_{n}\right\|=\operatorname{dist}\left(b_{n}, F\right)$. By the Attouch-Wets convergence of $\left\{A_{n}\right\}$ and $\left\{B_{n}\right\}$ to $A$ and $B$, respectively, there exist two sequences $\left\{x_{n}\right\}$ and $\left\{y_{n}\right\}$ such that $x_{n} \in A_{n}, y_{n} \in B_{n}(n \in \mathbb{N})$ and such that $\left\|x_{n}+v-f_{n}\right\| \rightarrow 0$ and $\left\|y_{n+1}-v-e_{n}\right\| \rightarrow 0$. Moreover, without any loss of generality we can suppose that $x_{n} \in E+\varepsilon B_{X}$ and $y_{n} \in F+\varepsilon B_{X}$, whenever $n \geq n_{1}$. Now, take $\eta^{\prime} \in(\eta, 1)$ and $\delta \in(0,1)$ satisfying $\frac{\delta+\eta}{1-\delta} \leq \eta^{\prime}$. By applying Proposition 4.6 there exists $n_{2} \geq n_{1}$ such that, for each $n \geq n_{2}$, we have:

(iii) if $\operatorname{dist}\left(a_{n}, E\right) \geq 2 \varepsilon$ and $\operatorname{dist}\left(b_{n}, F\right) \geq 2 \varepsilon$ then

$$
\cos \left(x_{n}-a_{n}, b_{n}-\left(a_{n}+v\right)\right) \leq \delta ;
$$

(iv) if $\operatorname{dist}\left(a_{n}, E\right) \geq 2 \varepsilon$ and $\operatorname{dist}\left(b_{n+1}, F\right) \geq 2 \varepsilon$ then

$$
\cos \left(y_{n+1}-b_{n+1}, a_{n}-\left(b_{n+1}-v\right)\right) \leq \delta .
$$

Taking into account (i)-(iv) and Fact 4.7, if $n \geq n_{2}$ then the following conditions hold:

- $\quad$ if $\operatorname{dist}\left(a_{n}, E\right) \geq 2 \varepsilon$ and $\operatorname{dist}\left(b_{n}, F\right) \geq 2 \varepsilon$ then

$$
\left\|a_{n}-x_{n}\right\| \leq \eta^{\prime}\left\|b_{n}-\left(x_{n}+v\right)\right\| ;
$$

- if $\operatorname{dist}\left(a_{n}, E\right) \geq 2 \varepsilon$ and $\operatorname{dist}\left(b_{n+1}, F\right) \geq 2 \varepsilon$ then

$$
\left\|b_{n+1}-y_{n+1}\right\| \leq \eta^{\prime}\left\|a_{n}-\left(y_{n+1}-v\right)\right\| .
$$

Now, by the triangle inequality, we have that, for each $n \geq n_{2}$, the following conditions hold (we provide all steps only in the first condition, since the second one follows similarly):

- $\quad$ if $\operatorname{dist}\left(a_{n}, E\right) \geq 2 \varepsilon$ and $\operatorname{dist}\left(b_{n}, F\right) \geq 2 \varepsilon$ then

$$
\begin{aligned}
\operatorname{dist}\left(a_{n}, E\right) & \leq\left\|a_{n}-\left(f_{n}-v\right)\right\| \\
& \leq\left\|a_{n}-x_{n}\right\|+\left\|x_{n}+v-f_{n}\right\| \\
& \leq \eta^{\prime}\left\|b_{n}-x_{n}-v\right\|+\left\|x_{n}+v-f_{n}\right\| \\
& \leq \eta^{\prime}\left\|b_{n}-x_{n}-v+f_{n}-f_{n}\right\|+\left\|x_{n}+v-f_{n}\right\| \\
& \leq \eta^{\prime}\left(\left\|b_{n}-f_{n}\right\|+\left\|x_{n}+v-f_{n}\right\|\right)+\left\|x_{n}+v-f_{n}\right\| ;
\end{aligned}
$$

- if $\operatorname{dist}\left(a_{n}, E\right) \geq 2 \varepsilon$ and $\operatorname{dist}\left(b_{n+1}, F\right) \geq 2 \varepsilon$ then

$$
\begin{aligned}
\operatorname{dist}\left(b_{n+1}, F\right) & \leq\left\|b_{n+1}-\left(e_{n}+v\right)\right\| \\
& \leq \eta^{\prime}\left(\left\|a_{n}-e_{n}\right\|+\left\|y_{n+1}-v-e_{n}\right\|\right)+\left\|y_{n+1}-v-e_{n}\right\| .
\end{aligned}
$$

If we consider $\eta^{\prime \prime} \in\left(\eta^{\prime}, 1\right)$, since $\left\|x_{n}+v-f_{n}\right\| \rightarrow 0$ and $\left\|y_{n+1}-v-e_{n}\right\| \rightarrow 0$, there exists $n_{3} \geq n_{2}$ such that if $n \geq n_{3}$ then the following conditions hold: 
$\left(\beta_{1}\right) \quad$ if $\operatorname{dist}\left(b_{n}, F\right) \geq 2 \varepsilon$ and $\operatorname{dist}\left(a_{n}, E\right) \geq 2 \varepsilon$ then

$$
\operatorname{dist}\left(a_{n}, E\right) \leq \eta^{\prime \prime} \operatorname{dist}\left(b_{n}, F\right) ;
$$

$\left(\beta_{2}\right) \quad$ if $\operatorname{dist}\left(a_{n}, E\right) \geq 2 \varepsilon$ and $\operatorname{dist}\left(b_{n+1}, F\right) \geq 2 \varepsilon$ then

$$
\operatorname{dist}\left(b_{n+1}, F\right) \leq \eta^{\prime \prime} \operatorname{dist}\left(a_{n}, E\right) .
$$

Now, there exists $n_{4} \geq n_{3}$ such that $\operatorname{dist}\left(a_{n_{4}}, E\right) \leq 2 \varepsilon$ or $\operatorname{dist}\left(b_{n_{4}}, F\right) \leq 2 \varepsilon$. Indeed, since $\eta^{\prime \prime}<1$, the fact that

$$
\operatorname{dist}\left(a_{n}, E\right) \geq 2 \varepsilon \text { and } \operatorname{dist}\left(b_{n}, F\right) \geq 2 \varepsilon, \quad \text { whenever } n \geq n_{3},
$$

contradicts the fact that $\left(\beta_{1}\right)$ and $\left(\beta_{2}\right)$ are satisfied whenever $n \geq n_{3}$. We conclude our proof by showing that $\operatorname{dist}\left(a_{n}\right) \leq M$ for every $n>n_{4}$. By contradiction, let us suppose that the set

$$
\Omega=\left\{n \in \mathbb{N}: n>n_{4} \text { and } \operatorname{dist}\left(a_{n}, E\right)>M\right\}
$$

is nonempty. Let $n_{5}=\min \Omega$. Then by $\left(\alpha_{1}\right)$, we have $\operatorname{dist}\left(b_{n_{5}}, F\right)>2 \varepsilon$. Moreover, by $\left(\alpha_{2}\right)$, it holds $\operatorname{dist}\left(a_{n_{5}-1}, E\right)>2 \varepsilon$. Now, from $\left(\beta_{2}\right)$, it follows that

$$
\operatorname{dist}\left(b_{n_{5}}, F\right) \leq \eta^{\prime \prime} \operatorname{dist}\left(a_{n_{5}-1}, E\right)<M .
$$

Finally, since $\operatorname{dist}\left(a_{n_{5}}, E\right)>M>2 \varepsilon$ and $\operatorname{dist}\left(b_{n_{5}}, F\right)>2 \varepsilon$, we conclude from $\left(\beta_{1}\right)$ that it holds

$$
\operatorname{dist}\left(a_{n_{5}}, E\right) \leq \eta^{\prime \prime} \operatorname{dist}\left(b_{n_{5}}, F\right)<M,
$$

a contradiction.

If the intersection of $A$ and $B$ is nonempty, we obtain, as an immediate consequence of Theorem 4.9, the following result.

Corollary 4.10 Let $A, B$ be closed convex nonempty subsets of $X$ such that $A \cap B$ is bounded and nonempty. If the couple $(A, B)$ is regular then the perturbed alternating projections sequences $\left\{a_{n}\right\}$ and $\left\{b_{n}\right\}$ satisfy $\operatorname{dist}\left(a_{n}, A \cap B\right) \rightarrow 0$ and $\operatorname{dist}\left(b_{n}, A \cap B\right) \rightarrow 0$

We conclude this section by showing some relationships between the results of [9] and Theorem 4.9. First of all, we briefly recall the notions of strongly exposed point and strongly exposing functional. This notions, and the corresponding dual versions (see, e.g., [6, Definition 6.2]), play an important role in the theory of Banach spaces.

Definition 4.11 (see, e.g., [11, Definition 7.10]) Let $A$ be a nonempty subset of a normed space $Z$. A point $a \in A$ is called a strongly exposed point of $A$ if there exists a support functional $f \in Z^{*} \backslash\{0\}$ for $A$ at $a$ (i.e., $f(a)=\sup f(A)$ ), such that $x_{n} \rightarrow a$ for all sequences $\left\{x_{n}\right\}$ in $A$ such that $\lim _{n} f\left(x_{n}\right)=\sup f(A)$. In this case, we say that $f$ strongly exposes $A$ at $a$.

Remark 4.12 If $f$ strongly exposes $A$ at $a$ then $a$ is the unique point at which $f$ assumes its maximum value on $A$. Indeed, let us suppose on the contrary that there exists $b \in A \backslash\{a\}$ such that $f(b)=\sup f(A)$, then we get a contradiction taking $x_{n}=b$, whenever $n \in \mathbb{N}$.

Definition 4.13 (see, e.g., [13, Definition 1.3] or [8]) Let $A$ be a body in a normed space $Z$. We say that $x \in \partial A$ is an LUR (locally uniformly rotund) point of $A$ if for each $\varepsilon>0$ there exists $\delta>0$ such that if $y \in A$ and $\operatorname{dist}(\partial A,(x+y) / 2)<\delta$ then $\|x-y\|<\varepsilon$. We say that $A$ is an LUR body if each point in $\partial A$ is an LUR point of $A$. 
The notion of LUR norm is a natural generalization of uniform rotundity and plays an important role in the theory of Banach spaces (see, e.g., [11] for the definition of and the main results on LUR norms; see also [7, 8] for some recent results involving this notion). Moreover, it is easy to see that, in the case $X$ is finite-dimensional, a body is LUR iff it is strictly convex.

The following lemma shows that each LUR point is a strongly exposed point.

Lemma 4.14 (see e.g. [10, Lemma 4.3]) Let $A$ be a body in a normed space $Z$ and suppose that $a \in \partial A$ is an LUR point of $A$. Then, if $f \in S_{Z^{*}}$ is a support functional for $A$ in $a, f$ strongly exposes $A$ at a.

First, we show that a more general variant of the assumptions of one of the main results in [9], namely [9, Theorem 3.3], implies that the couple $(A, B)$ is regular. It is interesting to remark that here we consider also the case in which $A$ and $B$ do not intersect.

Proposition 4.15 Let $A, B$ be nonempty closed convex subsets of $X$. Let us suppose that there exist $e \in A \cap(B-v)$ and a linear continuous functional $x^{*} \in S_{X^{*}}$ such that

$$
\inf x^{*}(B-v)=x^{*}(e)=\sup x^{*}(A)
$$

and such that $x^{*}$ strongly exposes $A$ at e. Then the couple $(A, B)$ is regular.

Proof There is no loss of generality in assuming $e=0$. We claim that $E=\{0\}$. Indeed, if we suppose on the contrary that there exists $e^{\prime} \in E \backslash\{e\}$, then we would have $x^{*}(e)=$ $\sup x^{*}(A)$; a contradiction against Remark 4.12. Now, suppose on the contrary that $(A, B)$ is not regular. Therefore there exist sequences $\left\{x_{n}\right\} \subset X,\left\{a_{n}\right\} \subset A,\left\{b_{n}\right\} \subset B$, and a real number $\bar{\varepsilon}>0$ such that

$$
\operatorname{dist}\left(x_{n}, E\right)=\left\|x_{n}\right\|>\bar{\varepsilon},
$$

and such that

$$
\operatorname{dist}\left(x_{n}, A\right)=\left\|x_{n}-a_{n}\right\| \rightarrow 0, \quad \operatorname{dist}\left(x_{n}, B-v\right)=\left\|x_{n}-b_{n}+v\right\| \rightarrow 0 .
$$

Since inf $x^{*}(B-v)=0=\sup x^{*}(A)$, we have

$$
x^{*}\left(x_{n}\right)=x^{*}\left(x_{n}-a_{n}\right)+x^{*}\left(a_{n}\right) \leq\left\|x_{n}-a_{n}\right\|
$$

and

$$
x^{*}\left(x_{n}\right)=x^{*}\left(x_{n}-b_{n}+v\right)+x^{*}\left(b_{n}-v\right) \geq-\left\|x_{n}-b_{n}+v\right\| .
$$

By the previous two inequalities and (10), it holds $\lim _{n} x^{*}\left(x_{n}\right)=0$. Since $\left\|x_{n}-a_{n}\right\| \rightarrow 0$, we have $\lim _{n} x^{*}\left(a_{n}\right)=0$. Moreover, since $x^{*}$ strongly exposes $A$ at $e$, the last equality implies that $\left\|a_{n}\right\| \rightarrow 0$. Indeed, to see this it, is sufficient to apply the definition of strongly exposing functional and take into account that $\lim _{n} x^{*}\left(a_{n}\right)=\sup x^{*}(A)$. We conclude that $\left\|x_{n}\right\| \rightarrow 0$, contrary to (9).

By combining the previous proposition and Theorem 4.9, we obtain the following corollary generalizing [9, Theorem 3.3].

Corollary 4.16 Let $A, B$ be nonempty closed convex subsets of $X$. Let us suppose that there exist $e \in A \cap(B-v)$ and a linear continuous functional $x^{*} \in S_{X^{*}}$ such that

$$
\inf x^{*}(B-v)=x^{*}(e)=\sup x^{*}(A)
$$

and such that $x^{*}$ strongly exposes $A$ at e. Then, the couple $(A, B)$ is stable. 
Moreover, in [9], the authors proved the following sufficient condition for the stability of a couple $(A, B)$.

Theorem 4.17 [9, Theorem 4.2] Let $X$ be a Hilbert space and A, B nonempty closed convex subsets of $X$. Suppose that int $(A \cap B) \neq \emptyset$, then the couple $(A, B)$ is stable.

By combining Corollary 4.16 and Theorem 4.17, we obtain the following sufficient condition for the stability of the couple $(A, B)$ generalizing [9, Corollary 4.3, (ii)].

Corollary 4.18 Let $X$ be a Hilbert space, suppose that $A, B$ are bodies in $X$ and that $A$ is LUR. Then the couple $(A, B)$ is stable.

Proof If int $(A \cap B) \neq \emptyset$, the thesis follows by applying Theorem 4.17. If int $(A \cap B)=\emptyset$, since $A$ and $B$ are bodies, we have $\operatorname{int}(A) \cap B=\emptyset$. Since $A$ is LUR the intersection $A \cap(B-v)$ reduces to a singleton $\{e\}$. By the Hahn-Banach theorem, there exists a linear functional $x^{*} \in X^{*}$ such that

$$
\inf x^{*}(B-v)=x^{*}(e)=\sup x^{*}(A) .
$$

Since $A$ is an LUR body, by Lemma 4.14, we have that $x^{*}$ strongly exposes $A$ at $e$. We are now in position to apply Corollary 4.16 and conclude the proof.

Finally, we show that $[9$, Theorem 5.2], follows by Theorem 4.9.

Corollary 4.19 Let $U, V$ be closed subspaces of $X$ such that $U \cap V=\{0\}$ and $U+V$ is closed. Then the couple $(U, V)$ is stable.

Proof Since $U+V$ is closed, by [1, Corollary 4.5], the couple $(U, V)$ is regular. By Theorem 4.9, the couple $(U, V)$ is $d$-stable. Since $U \cap V$ is a singleton, the couple $(U, V)$ is stable.

\section{Final Remarks, Examples, and an Open Problem}

Known examples show that the hypothesis about regularity of the couple $(A, B)$, in Theorem 4.9, is necessary. To see this, it is indeed sufficient to consider any couple $(A, B)$ of sets such that $A \cap B$ is a singleton and such that, for a suitable starting point, the method of alternating projections does not converge (see [12] for such a couple of sets).

A natural question is whether, in the same theorem, the hypothesis about regularity of the couple $(A, B)$ can be replaced by the weaker hypothesis that "for any starting point the method of alternating projections converges". The answer to previous question is negative; indeed, in [9, Theorem 5.7], the authors provided an example of a couple $(A, B)$ of closed subspaces of a Hilbert space such that $A \cap B=\{0\}$ and such that the couple $(A, B)$ is not stable (and hence not $d$-stable since $A \cap B$ is a singleton). It is interesting to observe that, by the classical von Neumann result [15], the method of alternating projections converges for this couple of sets.

The next example shows that, if we consider closed convex sets $A, B \subset X$ such that $A \cap B$ is nonempty and bounded, the regularity of the couple $(A, B)$ does not imply in 
general that $(A, B)$ is stable. In particular, we cannot replace $d$-stability with stability in the statement of Theorem 4.9 .

Example 5.1 ([9, Example 4.4]) Let $X=\mathbb{R}^{2}$ and let us consider, for each $h \in \mathbb{N}$, the following subsets of $H$ :

$$
\begin{aligned}
A & =\operatorname{conv}\{(1,1),(-1,1),(1,0),(-1,0)\} ; \\
C_{2 h} & =\operatorname{conv}\left\{(1,1),(-1,1),\left(1, \frac{1}{h}\right),(-1,0)\right\} ; \\
C_{2 h-1} & =\operatorname{conv}\left\{(1,1),(-1,1),(1,0),\left(-1, \frac{1}{h}\right)\right\} ; \\
B & =\operatorname{conv}\{(1,-1),(-1,-1),(1,0),(-1,0)\} ; \\
D_{2 h} & =\operatorname{conv}\left\{(1,-1),(-1,-1),\left(1,-\frac{1}{h}\right),(-1,0)\right\} ; \\
D_{2 h-1} & =\operatorname{conv}\left\{(1,-1),(-1,-1),(1,0),\left(-1,-\frac{1}{h}\right)\right\} .
\end{aligned}
$$

Then the couple $(A, B)$ is regular but not stable.

Fore the sake of completeness we include a proof of the previous example.

Proof By [1, Theorem 3.9], the couple $(A, B)$ is regular. Let us prove that $(A, B)$ is not stable. It is easy to see that $C_{h} \rightarrow A$ and $D_{h} \rightarrow B$ for the Attouch-Wets convergence. We claim that the couple $(A, B)$ is not stable. To prove this, let us consider the starting point $z_{0}=(0,0)$ and observe that, if we consider the points $a_{k}^{1}=\left(P_{C_{1}} P_{D_{1}}\right)^{k} z_{0}$, then $a_{k}^{1} \rightarrow(1,0)$ and hence there exists $N_{1} \in \mathbb{N}$ such that

$$
\left\|a_{N_{1}}^{1}-(1,0)\right\|<\frac{1}{4} \text {. }
$$

Define $A_{n}=C_{1}$ and $B_{n}=D_{1}$ whenever $1 \leq n \leq N_{1}$. Similarly, if we consider the points $a_{k}^{2}=\left(P_{C_{2}} P_{D_{2}}\right)^{k} a_{N_{1}}^{1}$, then $a_{k}^{2} \rightarrow(-1,0)$ and hence there exists $N_{2} \in \mathbb{N}$ such that

$$
\left\|a_{N_{2}}^{2}-(-1,0)\right\|<\frac{1}{4} \text {. }
$$

Define $A_{n}=C_{2}$ and $B_{n}=D_{2}$ whenever $N_{1}+1 \leq n \leq N_{1}+N_{2}$. Then, proceeding inductively, it is easy to construct sequences $\left\{A_{n}\right\}$ and $\left\{B_{n}\right\}$ converging respectively to $A$ and $B$ for the Attouch-Wets convergence and such that the perturbed alternating projections sequences $\left\{a_{n}\right\}$ and $\left\{b_{n}\right\}$, w.r.t. $\left\{A_{n}\right\}$ and $\left\{B_{n}\right\}$ and with starting point $z_{0}$, do not converge.

Now, the following example shows that, even in finite dimension, the hypothesis concerning the boundedness of the sets $E, F$ cannot be dropped in the statement of Theorem 4.9.

Example 5.2 Let $A, B$ be the subsets of $\mathbb{R}^{3}$ defined by

$$
A=\left\{(x, y, z) \in \mathbb{R}^{3} ; z=0, y \geq 0\right\}, \quad B=\left\{(x, y, z) \in \mathbb{R}^{3} ; z=0\right\},
$$

then the following conditions hold:

(a) $A \cap B$ coincides with $A$ (and hence $(A, B)$ is regular);

(b) $A \cap B$ is not bounded;

(c) the couple $(A, B)$ is not $d$-stable.

The proof of (a) and (b) is trivial. To prove (c), we need the following lemma. 
Lemma 5.3 Let $A, B$ be defined as in Example 5.2. For each $n \in \mathbb{N}$ and $x_{0} \geq 1$, let $P_{n, x_{0}}^{1}, P_{n, x_{0}}^{2}, P_{n, x_{0}}^{3} \in \mathbb{R}^{3}$ be defined by

$$
P_{n, x_{0}}^{1}=\left(x_{0}+n x_{0},-1,0\right), \quad P_{n, x_{0}}^{2}=\left(x_{0}+n x_{0}+\frac{1}{n x_{0}}, 0,0\right), \quad P_{n, x_{0}}^{3}=\left(0, \frac{1}{n}, \frac{1}{n}\right) .
$$

Let $t_{n, x_{0}}$ be the line in $\mathbb{R}^{3}$ containing the point $P_{n, x_{0}}^{1}$ and $P_{n, x_{0}}^{3}$, and let $r_{n, x_{0}}$ be the ray in $\mathbb{R}^{3}$ with initial point $P_{n, x_{0}}^{1}$ and containing the points $P_{n, x_{0}}^{2}$. Let $A_{n, x_{0}}, B_{n, x_{0}}$ be the closed convex subsets of $\mathbb{R}^{3}$ defined by

$$
A_{n, x_{0}}=\operatorname{conv}\left(t_{n, x_{0}} \cup r_{n, x_{0}}\right), \quad B_{n, x_{0}}=\left\{(x, y, z) \in \mathbb{R}^{3} ; z=0\right\} .
$$

Then the following conditions hold.

(i) for each $N \in \mathbb{N}, \lim _{n} h_{N}\left(A_{n, x_{0}}, A\right)=0$, uniformly with respect to $x_{0} \geq 1$;

(ii) For each $n \in \mathbb{N}$ and $x_{0} \geq 1$, the alternating projections sequences, relative to the sets $A_{n, x_{0}}, B_{n, x_{0}}$ and starting point $\left(x_{0}, 0,0\right)$, converge to $P_{n, x_{0}}^{1}$.

Proof (i) Let $n \in \mathbb{N}, x_{0} \geq 1$, and let us denote by $\left\{e_{1}, e_{2}, e_{3}\right\}$ the canonical basis of $\mathbb{R}^{3}$. Let $D_{n, x_{0}}: \mathbb{R}^{3} \rightarrow \mathbb{R}^{3}$ be the rotation such that

$$
\begin{aligned}
D_{n, x_{0}}\left(e_{1}\right) & =\frac{P_{n, x_{0}}^{1}-P_{n, x_{0}}^{3}}{\left\|P_{n, x_{0}}^{1}-P_{n, x_{0}}^{3}\right\|}:=v_{n, x_{0}}, \\
D_{n, x_{0}}\left(e_{2}\right) & =\frac{P_{n, x_{0}}^{2}-P_{n, x_{0}}^{1}}{\left\|P_{n, x_{0}}^{2}-P_{n, x_{0}}^{1}\right\|}:=w_{n, x_{0}}, \\
D_{n, x_{0}}\left(e_{3}\right) & =v_{n, x_{0}} \wedge w_{n, x_{0}},
\end{aligned}
$$

where $v_{n, x_{0}} \wedge w_{n, x_{0}}$ denotes the standard vector product between $v_{n, x_{0}}$ and $w_{n, x_{0}}$ in $\mathbb{R}^{3}$. An elementary computation shows that $\left\|v_{n, x_{0}}-e_{1}\right\|,\left\|w_{n, x_{0}}-e_{2}\right\|$ (and hence $\| v_{n, x_{0}} \wedge w_{n, x_{0}}-$ $\left.e_{3} \|\right)$ go to 0 as $n \rightarrow \infty$, uniformly w.r.t. $x_{0} \geq 1$. This implies that $\left\|D_{n, x_{0}}-I\right\|$ goes to 0 as $n \rightarrow \infty$, uniformly w.r.t. $x_{0} \geq 1$. By definitions of $A_{n, x_{0}}$ and $A$, we have

$$
A_{n, x_{0}}=P_{n, x_{0}}^{3}+D_{n, x_{0}}(A) \text {. }
$$

Fix $N \in \mathbb{N}$. Fact 2.3 implies that $h_{N}\left(A_{n, x_{0}}, A\right)$ goes to 0 as $n \rightarrow \infty$, uniformly w.r.t. $x_{0} \geq 1$; the proof is concluded.

(ii) Observe that:

- the projection of the line $t_{n, x_{0}}$ onto the plane $B$ is the line $s_{n, x_{0}}$ containing $P_{n, x_{0}}^{1}$ and the point $\left(x_{0}, 0,0\right)$;

- the projection of the line $s_{n, x_{0}}$ onto the unique plane containing $A_{n, x_{0}}$ is the line $t_{n, x_{0}}$.

Hence, the alternating projections sequences, relative to the sets $A_{n, x_{0}}, B_{n, x_{0}}$ and starting point $\left(x_{0}, 0,0\right)$, converge to intersection point of the lines $t_{n, x_{0}}$ and $s_{n, x_{0}}$, i.e., $P_{n, x_{0}}^{1}$.

Proof of Example 5.2, (c) Fix the starting point $a_{0}=(1,0,0)$ and let $A_{1,1}, B_{1,1}, P_{1,1}^{1}$, be defined by Lemma 5.3. Observe that, if we consider the points

$$
a_{k}^{1}=\left(P_{A_{1,1}} P_{B_{1,1}}\right)^{k} a_{0} \quad(k \in \mathbb{N}),
$$

by Lemma 5.3, (ii), there exists $N_{1} \in \mathbb{N}$ such that $\operatorname{dist}\left(a_{N_{1}}^{1}, A \cap B\right) \geq \frac{1}{2}$, indeed, $\left\{a_{k}^{1}\right\}_{k}$ converges to the point $P_{1,1}^{1}=(2,-1,0)$ and $\operatorname{dist}\left(P_{1,1}^{1}, A \cap B\right)=1$. Define $A_{n}=A_{1,1}$ and $B_{n}=B_{1,1}=B$, whenever $1 \leq n \leq N_{1}$. Then define $A_{N_{1}+1}=A$ and $B_{N_{1}+1}=B$, and 
observe that $a_{0}^{2}:=P_{A} P_{B} a_{N_{1}}^{1}=\left(x_{1}, 0,0\right)$ for some $x_{1} \geq 1$. Proceeding as above, if we consider the points

$$
a_{k}^{2}=\left(P_{A_{2, x_{1}}} P_{B_{2, x_{1}}}\right)^{k} a_{0}^{2} \quad(k \in \mathbb{N}),
$$

then there exists $N_{2} \in \mathbb{N}$ such that $\operatorname{dist}\left(a_{N_{2}}^{2}, A \cap B\right) \geq \frac{1}{2}$. Define $A_{n}=A_{2, x_{1}}, B_{n}=B_{2, x_{1}}=$ $B$, whenever $N_{1}+1<n \leq N_{2}$. Then define $A_{N_{2}+1}=A, B_{N_{2}+1}=B$, and observe that $a_{0}^{3}:=P_{A} P_{B} a_{N_{2}}^{2}=\left(x_{2}, 0,0\right)$ for some $x_{2} \geq 1$. Then, proceeding inductively, we can construct sequences $\left\{A_{n}\right\}$ and $\left\{B_{n}\right\}$ such that, by Lemma 5.3, (i), $A_{n} \rightarrow A$ and $B_{n} \rightarrow B$ for the Attouch-Wets convergence. Moreover, by our construction, it is easy to see that the corresponding perturbed alternating projections sequences $\left\{a_{n}\right\}$ and $\left\{b_{n}\right\}$, with starting point $a_{0}$, are such that

$$
\lim \sup _{n} \operatorname{dist}\left(a_{n}, A \cap B\right) \geq \frac{1}{2} .
$$

This proves that the couple $(A, B)$ is not $d$-stable.

Finally, we conclude with an open problem asking whether the inverse of Theorem 4.9 holds true.

Problem 5.4 Let $A, B$ be closed convex nonempty subsets of $X$ such that $E$ and $F$ are nonempty and bounded. Suppose that the couple $(A, B)$ is $d$-stable. Does the couple $(A, B)$ is regular?

Acknowledgements The research of the authors is partially supported by GNAMPA-INdAM, Progetto GNAMPA 2020. The second author is also partially supported by the Ministerio de Ciencia, Innovación y Universidades (MCIU), Agencia Estatal de Investigación (AEI) (Spain) and Fondo Europeo de Desarrollo Regional (FEDER) under project PGC2018-096899- B-I00 (MCIU/AEI/FEDER, UE). Data sharing not applicable to this article as datasets were not generated or analysed during the current study.

Funding Open access funding provided by Università Cattolica del Sacro Cuore within the CRUI-CARE Agreement.

Open Access This article is licensed under a Creative Commons Attribution 4.0 International License, which permits use, sharing, adaptation, distribution and reproduction in any medium or format, as long as you give appropriate credit to the original author(s) and the source, provide a link to the Creative Commons licence, and indicate if changes were made. The images or other third party material in this article are included in the article's Creative Commons licence, unless indicated otherwise in a credit line to the material. If material is not included in the article's Creative Commons licence and your intended use is not permitted by statutory regulation or exceeds the permitted use, you will need to obtain permission directly from the copyright holder. To view a copy of this licence, visit http://creativecommons.org/licenses/by/4.0/.

\section{References}

1. Bauschke, H.H., Borwein, J.M.: On the convergence of von Neumann's alternating projection algorithm for two sets. Set-Valued Anal. 1, 185-212 (1993)

2. Beer, G.: Topologies on Closed and Closed Convex Sets, Mathematics and its Applications, vol. 268. Kluwer Academic Publishers Group, Dordrecht (1993)

3. Borwein, J.M., Sims, B., Tam, M.K.: Norm convergence of realistic projection and reflection methods. Optimization 64, 161-178 (2015)

4. Borwein, J.M., Zhu, Q.J.: Techniques of Variational Analysis, CMS Books in Mathematics/Ouvrages de Mathématiques de la SMC. Springer, New York (2005)

5. Brègman, L.M.: Finding the common point of convex sets by the method of successive projection. Dokl. Akad. Nauk SSSR 162(1965), 487-490 (1965)

6. De Bernardi, C.A., Veselý, L.: Intersection properties of the unit ball. J. Math. Anal. Appl. 475, 11081129 (2019) 
7. De Bernardi, C.A., Somaglia, J., Veselý, L.: Star-finite coverings of Banach spaces. J. Math. Anal. Appl. 491, 124384, 21 (2020)

8. De Bernardi, C.A., Veselý, L.: Tilings of normed spaces. Canad. J. Math. 69, 321-337 (2017)

9. De Bernardi, C.A., Miglierina, E.: A variational approach to the alternating projections method. J. Global Optim. https://doi.org/10.1007/s10898-021-01025-y (2021)

10. De Bernardi, C.A., Miglierina, E., Molho, E.: Stability of a convex feasibility problem. J. Global Optim. 75, 1061-1077 (2019)

11. Fabian, M., Habala, P., Hájek, P., Montesinos, V., Zizler, V.: Banach Space Theory. The Basis for Linear and Nonlinear Analysis, CMS Books in Mathematics/Ouvrages de Mathématiques de la SMC. Springer, New York (2011)

12. Hundal, H.S.: An alternating projection that does not converge in norm. Nonlinear Anal. 57, 35-61 (2004)

13. Klee, V., Veselý, L., Zanco, C.: Rotundity and smoothness of convex bodies in reflexive and nonreflexive spaces. Studia Math. 120, 191-204 (1996)

14. Lucchetti, R.: Convexity and Well-Posed Problems, CMS Books in Mathematics/Ouvrages de Mathématiques de la SMC. Springer, New York (2006)

15. von Neumann, J.: On rings of operators. Reduction theory. Ann. of Math. 50, 401-485 (1949)

Publisher's Note Springer Nature remains neutral with regard to jurisdictional claims in published maps and institutional affiliations. 